\title{
High-sensitivity detection of NO in a flame using a tunable ArF laser
}

\author{
A. M. Wodtke, L. Huwel, H. Schluter, G. Meijer, and P. Andersen \\ Max-Planck-Institut für Strömungsforschung, Bunsenstrasse 10, D-3400 Göttingen, Federal Republic of Germany \\ H. Voges \\ Laser Laboratorium Göttingen e.V., Tamannstrasse 6, D-3400 Göttingen, Federal Republic of Germany
}

Received May 4, 1988; accepted June 27, 1988

\begin{abstract}
A tunable, narrow-band $\mathrm{ArF}$ laser has been used for laser-induced fluorescence detection of NO in natural abundance in a flame experiment. $P$ and $R$ branches of the $D^{2} \Sigma v^{\prime}=0 \leftarrow X^{2} \Pi_{3 / 2,1 / 2} v^{\prime \prime}=1$ transition were observed probing rotational states between $J^{\prime \prime}=19.5$ and $J^{\prime \prime}=44.5$. A single-shot detection limit of 1 part in $10^{6}$ was found with a monochromator-based, dispersed-fluorescence detection system. In an experimental setup, determination of undispersed laser-induced fluorescence detection limits at or below the 1-part-in- $10^{9}$ range should be possible, because the narrow-band laser can be used to suppress all other sources of contaminating fluorescence even for detection of trace NO. The NO $B^{2} \Pi v^{\prime}=7 \leftarrow X^{2} \Pi v^{\prime \prime}=0$ transition was also observed in a cell experiment but not in the flame and is reported here.
\end{abstract}

Because of the role of $\mathrm{NO}$ as a pollutant, the detection of NO in combustion systems is a topic of considerable applied importance. Laser detection techniques have been widely employed. ${ }^{1}$ Coherent anti-Stokes Raman spectroscopy has had great success in the detection of majority constituents, but since the abundance of NO is typically on the parts-in- $10^{6}$ (ppm) level, other techniques must be sought. The use of laserinduced fluorescence (LIF) provides a sensitive and nonintrusive detection method for such purposes. NO has been detected with LIF in NO-doped flame experiments using the $A \leftarrow X$ gamma band at $226 \mathrm{~nm}$, suggesting detection limits of $20-30 \mathrm{ppm} .^{2-5}$ The advent of the beta-barium borate doubling crystal should improve this by at least an order of magnitude for small probe volumes in a flame. Nonetheless, the relatively long fluorescence lifetime of the NO $A$ state (250 nsec) leads to a further loss of sensitivity because of quenching.

Tunable excimer lasers have been shown to be ideal light sources for the analysis of combustion systems.,7 By using a tunable ArF laser with a monochromatorbased dispersed-fluorescence apparatus, the 1-ppm detection limit was achieved. NO in the flame was easily detected without doping. With this laser the $D^{2} \Sigma v^{\prime}=0 \leftarrow X^{2} \Pi_{3 / 2,1 / 2} v^{\prime \prime}=1$ transition in $\mathrm{NO}$ can be saturated with unfocused light, and because of its short fluorescence lifetime ( $20 \mathrm{nsec}$ ), quenching is less severe than in the $A$ state. Dispersed-fluorescence experiments show that trace NO can be selectively detected with the narrow-band laser. Therefore use of a high-transmission broadband filter and high-efficiency collection optics should yield a single-shot detection limit at or below 1 part in $10^{9}(\mathrm{ppb})$.

The experimental arrangement was described previously. ${ }^{6}$ The unfocused output $(1 \mathrm{~cm} \times 3 \mathrm{~cm})$ of a tunable ArF laser (Lambdaphysik EMG150 or EMG16OMSC) was used to excite NO fluorescence in the flame of a conventional propane soldering torch. The laser had a band width of $1.0 \mathrm{~cm}^{-1}$ (EMG150) or 0.5 $\mathrm{cm}^{-1}$ (EMG160MSC) and was operated at a pulse energy of $100 \mathrm{~mJ}$. The pulse duration is specified by Lambdaphysik to be 10-15 nsec. The locking efficiency was greater than $90 \%$ over most of the scanning range of both lasers. One part in $10^{3}$ of the fluorescence was collected with $f / 5.3$ optics and was dispersed through a MacPherson 218 0.3-m monochromator equipped with a $1200-\mathrm{mm}^{-1}$ grating, producing a linear dispersion of $2.65 \mathrm{~nm} / \mathrm{mm}$. Typically, spectra were obtained with $600-\mu \mathrm{m}$ slits. The sampling volume in the flame was determined by the apertures of the monochromator and was estimated to be $0.6 \mathrm{~cm} \times$ $0.06 \mathrm{~cm} \times 1.0 \mathrm{~cm}$. Detection was made with an RCA C31034 photomultiplier tube, and signal averaging was performed with a boxcar integrator. The spectra shown below were averaged over three shots of the laser. The cell experiments were performed at room temperature with pure-NO pressures from 200 m'Torr to 2 Torr.

Figure 1 shows the flame LIF spectrum of naturally occurring NO observed when the monochromator is adjusted to monitor the 208.0-nm emission of the $D$ state, $v^{\prime}=0 \rightarrow v^{\prime \prime}=3$. All lines can be unambiguously assigned to $P$ and $R$ branches of the $D^{2} \Sigma v^{\prime}=0 \leftarrow$ $X^{2} \Pi_{3 / 2,1 / 2} v^{\prime \prime}=1$ transition of NO. The LIF spectra in Fig. 2 were taken in a cell at 2-Torr pressure and $300 \mathrm{~K}$. The strongest features at room temperature are due to the $B^{2} \Pi v^{\prime}=7 \leftarrow X^{2} \Pi v^{\prime \prime}=0$ transition, which probes states with concentrations approximately 200 times higher. Both the $B \leftarrow X$ transition in the cell experiment and the $D \leftarrow X$ transition in the flame were linearly dependent on laser power up to $2 \mathrm{MW} / \mathrm{cm}^{2}$ 

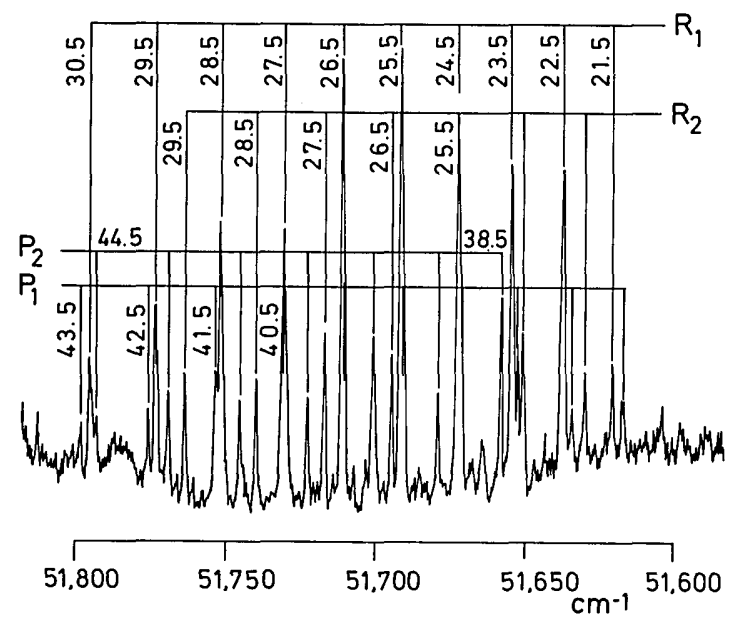

Fig. 1. NO $D^{2} \Sigma v^{\prime}=0 \leftarrow X^{2} \Pi_{3 / 2,1 / 2} v^{\prime \prime}=1$ transitions observed with an unfocused tunable ArF laser in a propane$\mathrm{O}_{2}$ flame. The concentration of $\mathrm{NO}$ is estimated to be 100 ppm. Emission from $v^{\prime}=0$ to $v^{\prime \prime}=3$ was monitored at 208.0 $\mathrm{nm}$ with a 0.6-nm monochromator resolution.

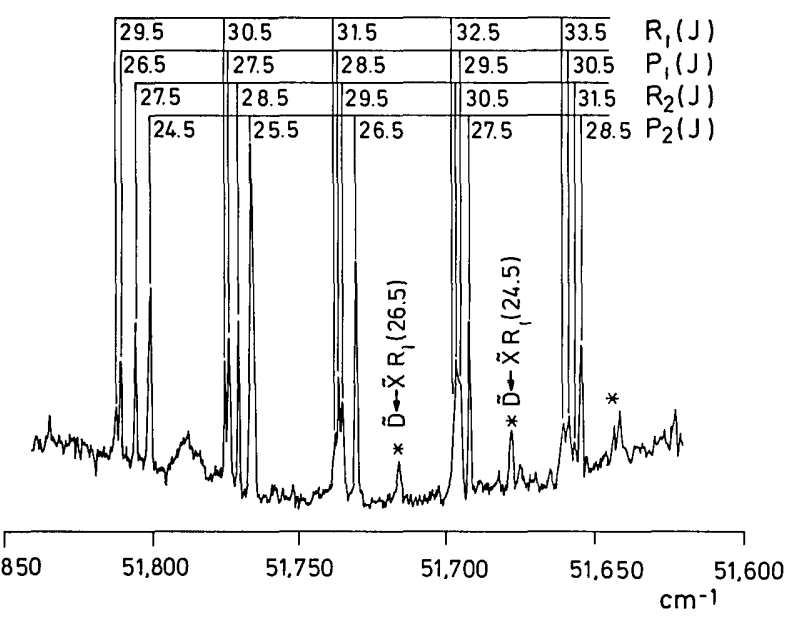

Fig. 2. NO $D^{2} \Sigma v^{\prime}=0 \leftarrow X^{2} \Pi_{3 / 2,1 / 2} v^{\prime \prime}=1$ (asterisks) and $B^{2} \Pi v^{\prime}=7 \leftarrow X^{2} \Pi v^{\prime \prime}=0$ transitions measured in a cell at 2 Torr pressure and $300 \mathrm{~K}$. Monochromator conditions are the same as for Fig. 1.

$\mathrm{cm}^{-1}$, where saturation of the transitions was observed. In Fig. 2 the $D \leftarrow X$ transition is still clearly seen at room temperature, despite the fact that it is originating in states $2900 \mathrm{~cm}^{-1}$ above the lowest quantum state of NO. Indeed, the $R_{1}(26.5)$ transition is observable with single laser shots down to $200 \mathrm{~m}$ Torr in the cell experiment. This permits a good estimation of the detection sensitivity in the flame experiment.

From previous experiments it was determined that in the region of the flame probed by the monochromator the temperature is of the order of $1750 \pm 250 \mathrm{~K} .{ }^{7}$ At this temperature the population where $v^{\prime \prime}=1$ and $J^{\prime \prime}=26.5$, probed by the $R_{1}(26.5)$ transition, is increased by $2.5 \times 10^{4}$ over that in the cell experiment. Neglecting fluorescence quenching for the moment, we would say that $8 \times 10^{-6}$ Torr $\mathrm{NO}$, or $10 \mathrm{ppb}$, could then be detected in the flame. The quantitative evaluation of quenching in flames is a difficult problem, and, in the absence of the needed quenching-rate constant data, we have assumed that the major quenching of the $D$ state is due to collisions with $\mathrm{N}_{2}$ and that the quenching-rate constants derived for $300 \mathrm{~K}$ can be applied. ${ }^{8}$ The quenching rates derived in this way are consistent with many data that indicate quenching rates of $10^{9}-10^{10} \mathrm{sec}^{-1}$ for open atmospheric flames. ${ }^{9}$ This gives a fluorescence loss of $10^{-2}$, meaning that the detection limit is actually of the order of $1 \mathrm{ppm}$ with the current experimental arrangement. From the absolute magnitude of the signal it was estimated that the observed concentration of NO is of the order of 100 ppm, consistent with other observations. ${ }^{2}$ The absence of the $B \leftarrow X$ transition in the flame is explained by a calculation similar to that just described, which predicts a detection limit of $50-100 \mathrm{ppm}$.

The 1-ppm detection limit observed for the $D \leftarrow X$ transition can be greatly improved, as discussed below. The greatest loss in experimental sensitivity is due to the use of a monochromator, which collects only $10^{-3}$ of the fluorescence and has a transmission efficiency of 0.1. The selective detection of flame species often requires the use of dispersed-fluorescence techniques, because in the complex environment of a flame there are many possible absorbers and fluorescers other than the molecule of interest. This is especially true when one is detecting minority species at the ppm level. If the molecule of interest can be selectively excited by the laser, a large increase in detection sensitivity can be achieved.

In particular, with an ArF laser there is a problem because of the numerous $\mathrm{O}_{2}$ transitions that are observed with strong LIF signals despite the fast predissociation of the excited electronic state. ${ }^{7}$ This can be clearly seen from Fig. 3(a), which shows the dispersedfluorescence spectrum observed when a broadband ArF laser is employed. $\mathrm{O}_{2}$ accounts for more than $50 \%$ of the fluorescence. Without the use of a monochromator, $\mathrm{NO}$ cannot be distinguished from $\mathrm{O}_{2}$ with the broadband laser.

Figure 3(b) shows the dispersed-fluorescence spectrum observed when the $R_{1}(26.5)$ transition is excited with a narrow-band $\mathrm{ArF}$ laser. Two progressions are observed, one from the $D$ state $\left(v^{\prime}=0 \rightarrow v^{\prime \prime}=2-5\right)$ and one due to rapid quenching of the $D$ state to the $C$ state and subsequent fluorescence $\left(v^{\prime}=0 \rightarrow v^{\prime \prime}=1-4\right)$. The $C$-state progression is shifted $919 \mathrm{~cm}^{-1}$ to the red and has the same Franck-Condon factor pattern as the $D$ state, an expected result owing to the similarity of the $D$ and $C$ states. Laser excitation spectra at an emission wavelength of $221.2 \mathrm{~nm}$ yield the same $D \leftarrow X$ spectra as in Fig. 1, confirming that quenching of the $D$ state is responsible for the $C$-state emission and that no direct excitation of the $C$ state is observed.

The resolution of the monochromator was set high enough to observe the single $P, R$ line pair emission, expected if $\mathrm{O}_{2}$ were excited by an accidental coincidence with the narrow-band component of the laser output. Since this is not observed, any $\mathrm{O}_{2}$ fluorescence can be excited only by the broadband component of the laser, which is at least 10 times smaller in 


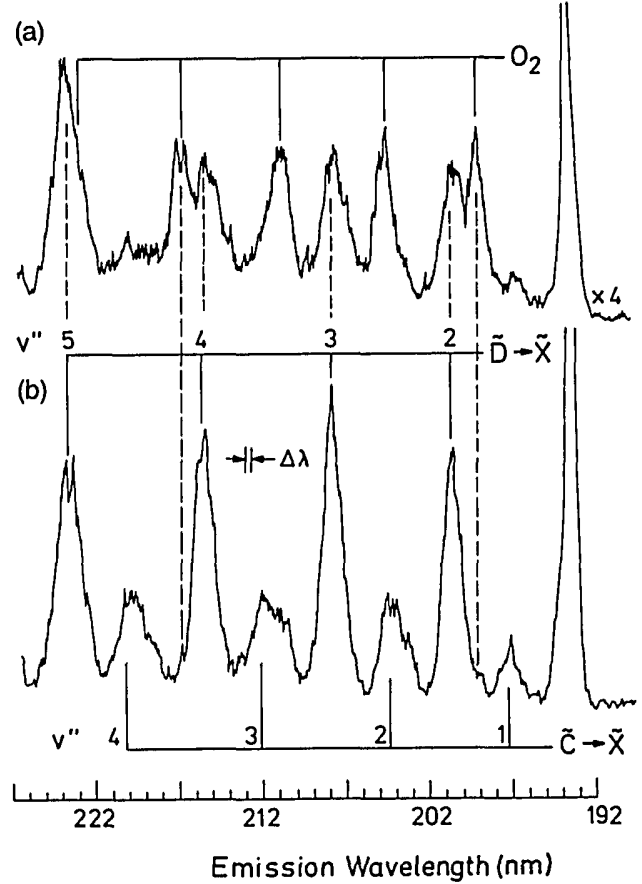

Fig. 3. (a) Emission spectrum observed with broadband laser excitation in the flame. (b) Emission spectrum observed when the $D^{2} \Sigma v^{\prime}=0 \leftarrow X^{2} \Pi_{3 / 2,1 / 2} v^{\prime \prime}=1, R_{1}$ (26.5) transition is excited with the narrow-band laser. Quenching of the NO $C$ state is observed, and $\mathrm{O}_{2}$ fluorescence is suppressed by at least a factor of 40 .

the spectrum of Fig. 3(b) than in the spectrum of Fig. $3(\mathrm{a})$, for which the laser was operated completely broadband. This means that $\mathrm{O}_{2}$ fluorescence is suppressed to less than $1.3 \%$ of the total fluorescence. This shows the power of the narrow-band tunable excimer laser, which in this case suppresses contaminating fluorescence due to $\mathrm{O}_{2}$, present at more than $10^{4}$ higher concentrations, and delivers spectrally clean information on a trace species in the flame.

Because the narrow-band properties of the laser can be used to excite NO selectively, it should be possible to perform NO detection with high-transmission filters and low-f-number collection optics. The gain in sensitivity with such a setup is of the order of $10^{3}$ compared with what is possible with the monochromator. It is anticipated that, with such an experimental arrangement, NO will be detectable with single laser shots at or below the 1-ppb level in a real combustion system. NO LIF imaging without doping should also be achievable with single laser shots.

LIF detection of NO in natural abundance in a flame has been observed with a high signal-to-noise ratio by using a narrow-band tunable ArF laser, an ideal tool for combustion analysis. A detection limit of $1 \mathrm{ppm}$ was obtained with a monochromator-based dispersed-fluorescence detection method, in which only $10^{-4}$ of the fluorescence light is observed by the photomultiplier. Selective excitation of NO with the narrow-band laser yields a clean NO emission spectrum over a large spectral range and presents the possibility of using high-transmission filters with highefficiency collection optics to bring the detection limit to the 1-ppb level. Two-dimensional imaging of NO without seeding should also be possible.

\section{References}

1. S. S. Penner, C. P. Wang, and M. Y. Bahadori, in Proceedings of the Twentieth International Symposium on Combustion (Combustion Institute, Pittsburgh, Pa., 1984), p. 1149.

2. D. R. Grieser and R. H. Barnes, Appl. Opt. 19, 741 (1980).

3. J. F. Verdieck and P. A. Bonczyk, in Proceedings of the Eighteenth International Symposium on Combustion (Combustion Institute, Pittsburgh, Pa., 1981), p. 1559.

4. C. Morely, in Proceedings of the Eighteenth International Symposium on Combustion (Combustion Institute, Pittsburgh, Pa., 1981), p. 23.

5. G. Kychakoff, K. Knapp, R. P. Howe, and R. K. Hanson, AIAA J. 22, 153 (1984).

6. P. Andresen, A. Bath, W. Groeger, H. W. Luelf, G. Meijer, and J. J. ter Meulen, Appl. Opt. 27, 365 (1988).

7. A. M. Wodtke, L. Huwel, H. Schluter, H. Voges, G. Meijer, and P. Andresen, in Proceedings of the Twentyfourth International Symposium on Combustion (Combustion Institute, Pittsburgh, $\mathrm{Pa}$., to be published).

8. H. Okabe, Photochemistry of Small Molecules (Wiley, New York, 1978), p. 174.

9. R. P. Lucht, in Laser Spectroscopy and Its Applications, L. J. Radziemski, R. W. Solarz, and J. A. Paisner, eds., Vol. 11 of Optical Engineering Series (Dekker, New York, 1987), p. 623. 